\title{
COGNIÇÃO, MOTRICIDADE, AUTOCUIDADOS, LINGUAGEM E SOCIALIZAÇÃO NO DESENVOLVIMENTO DE CRIANÇAS EM CRECHE
}

\author{
COGNITION, MOTOR ACTIVITY, SELF CARE, LANGUAGE \\ AND SOCIALIZATION DURING CHILDREN \\ DEVELOPMENT IN DAY CARE
}

Agnes Maria Gomes Murta $^{1}$
Angelina do Carmo Lessa $^{1}$
Antônio Sousa Santos $^{1}$
Nadja Maria Gomes Murta $^{1}$
Rosana Passos Cambraia $^{1}$

Murta AMG et al. Cognição, motricidade, autocuidados, linguagem e socialização no desenvolvimento de crianças em creche. Rev Bras Cresc e Desenv Hum 2011; 21(2): 220-229.

\section{RESUMO}

Objetivo: avaliar o desenvolvimento cognitivo e motor, autocuidados, linguagem, socialização de crianças e estado nutricional de zero a seis anos. Método: como sujeitos, participaram 48 crianças matriculadas na instituição filantrópica Casa da Criança, residentes na periferia da cidade de Diamantina (Vale do Jequitinhonha, Minas Gerais, Brasil). Para a coleta dos dados foram utilizados os seguintes instrumentos e procedimentos: aplicação do Inventário Portage, avaliação nutricional e aplicação de questionário sócio-econômico. Resultados: a análise de correlação parcial revelou que ocorreu correlação estatisticamente significante entre os pares de variáveis: cognição x linguagem, socialização $\mathrm{x}$ autocuidado e motor x peso/altura e peso/idade. Discussão: percebeu-se a necessidade de implantação de um programa nas creches na área de desenvolvimento infantil, que integre saúde e educação, e que possa ser planejado a partir da perspectiva do Inventário Portage. A utilização das ferramentas como as definidas no estudo possibilita a otimização dos repertórios das crianças, a capacitação dos educadores e a orientação dos familiares para estimulação das crianças. Conclusão: uma intervenção baseada no Inventário Portage mostra-se promissora para promoção das interações saudáveis entre as crianças e pais/cuidadores.

Palavras-chave: atividade motora; cognição; desenvolvimento infantil; linguagem infantil; nutrição infantil; socialização.

1 Professores da Faculdade de Ciências Biológicas e da Saúde - FCBS, Universidade Federal dos Vales do Jequitinhonha e Mucuri - UFVJM, Campus Diamantina, MG, Brasil. Correspondência: Rosana P. Cambraia, Grupo Jequi Saúde Coletiva, Faculdade de Ciências Biológicas e da Saúde - FCBS, Universidade Federal dos Vales do Jequitinhonha e Mucuri - UFVJM. Rua da Glória 187, sala 15, Centro, Diamantina, MG, 39100-000. Telefone: 38 3532-6054. Email: rosacambraia@pq.cnpq.br Apoio: Conselho Nacional de Desenvolvimento Científico e Tecnológico (CNPq), Edital Ciências Humanas 06/2003, processo $\mathrm{n}^{\circ} 403.806 / 2003-8$. 


\begin{abstract}
Objective: to evaluate the cognition and motor development, self care, language, socialization and the nutritional status of child with zero to six years old. Method: 48 children attending a philanthropic day care called Casa da Criança, living in Diamantina Town (Jequitinhonha Valley, Minas Gerais, Brazil) participated in this study. For data collection it was used the following instruments and proceedings: Portage Inventory application, nutritional evaluation and social and economic questionnaire. Results: the partial correlation analyses demonstrated that there were significant correlations within the following pair of variables: cognition $\mathrm{x}$ language, socialization $\mathrm{x}$ self care and motor $\mathrm{x}$ weight/height and weight/age. Discussion: we perceived the necessity of implantation of a program for child development stimulation in the day care that integrate health and education, which may be planned into the Portage Inventory perspective. The use of instruments in this study makes possible the children repertory optimization, the educators training and the family orientation to the children stimulation. Conclusion: an intervention based on the Portage Inventory shows as promising tool to the healthy interaction between the children and parents/caregivers.
\end{abstract}

Key words: motor activity; cognition; child development; child language; infant nutrition; socialization

\section{INTRODUÇÃO}

A situação da miséria e da fome reflete a longa história de exclusão social e econômica de parcelas significativas da população brasileira, especialmente no que se refere ao acesso à educação, à informação e aos recursos produtivos ou empregos que garantam a construção de um mínimo de autonomia para o ser humano ${ }^{1}$. Sobre o estado nutricional, alguns pontos críticos devem ser considerados, como: período crítico de deficiência nutricional e alimentar, interação de múltiplos nutrientes, variáveis como fisiologia, ambiente e fatores sociais ${ }^{2}$.

A desnutrição é resultado de complexa interação de fatores que envolvem diferentes aspectos, entre os quais se podem citar: a produção de alimentos, o acesso e o consumo dos gêneros alimentícios entre os membros da família, os tipos de cuidados disponíveis para a mulher e a criança, a disponibilidade de água limpa e saneamento, o acesso a serviços básicos de saúde e alimentação escolar.

A desnutrição pode assumir formas variadas que frequentemente se manifestam jun- tas, reforçando-se mutuamente, como a desnutrição protéico-calórica e as deficiências de microminerais, como o ferro ${ }^{3,4}$, o zinco ${ }^{5,6} \mathrm{e}$ as vitaminas ${ }^{7}$. Além disso, a desnutrição envolve também outros elementos de origem fisiológica, social, política, econômica e cultural. A relação entre a educação materna e o estado nutricional da criança vem sendo investigada com mais atenção, com enfoque integrado de saúde e comportamento ${ }^{8,9}$.

A questão vai além da sobrevivência infantil e da mortalidade e da morbidade maternas. Além de suportar incapacitação e fragilidade em seu sistema imunológico, as crianças malnutridas sofrem ainda de limitação em sua capacidade de aprendizagem ${ }^{10,11}$. Em crianças, a desnutrição desmotiva a curiosidade e reduz as atividades ligadas aos atos de brincar e explorar. Esses efeitos comprometem o desenvolvimento mental e cognitivo, reduzindo os níveis de interação da criança tanto com o ambiente quanto com as pessoas responsáveis por ela ${ }^{12,13}$.

Um estudo ${ }^{14}$ sobre os efeitos de um programa de suplementação nutricional durante 
dois anos revelou as consequências da suplementação com ou sem a estimulação do desenvolvimento psicomotor de crianças malnutridas. Quatro anos após o fim da intervenção as crianças apresentaram benefícios no fator percepção-motricidade. Também em outra pesquisa ${ }^{15}$ semelhante, verificou-se que baixa estatura no início da vida da criança está associada a uma série de defasagens na função cognitiva e na educação escolar. Enfim, a desnutrição no início da vida modifica a trajetória de desenvolvimento da criança, a menos que haja reabilitação em médio e longo prazos.

$\mathrm{Na}$ América Latina, as condições que propiciam a redução da mortalidade infantil estão relacionadas às boas práticas de cuidados e ao acesso aos serviços básicos de saúde, incluindo planejamento familiar e serviços de água e saneamento. As condições básicas para esse progresso envolvem a capacitação da mulher, traduzida em educação e controle dos recursos monetários ${ }^{12}$. Além da questão da mortalidade, os estudos envolvendo a qualidade do desenvolvimento infantil ainda precisam apontar, entre as populações em situação de risco, qual realmente é o prejuízo que advém da baixa capacidade intelectual em condições adversas e quais as populações especialmente vulneráveis.

O desenvolvimento infantil tem sido avaliado por um instrumento conhecido como Guia Portage de Educação Pré-Escolar ${ }^{16}$, que faz parte de um sistema de treinamento de pais e educadores do nível pré-escolar. Tal projeto teve início em 1969 em Portage, Wisconsin (Estados Unidos da América), visando desenvolver e implementar um programa modelo que atendesse crianças em fase pré-escolar com dificuldades de desenvolvimento. A escala avalia cinco áreas (cognitiva, motora, autocuidados, linguagem e socialização), totalizando 580 itens de avaliação. Pode ser utilizado tanto com crianças normais (zero a seis anos), para determinar o nível de desenvolvimento, quanto com crianças com algum tipo de dificuldade e impedimento, para determinar seu desenvolvimento e planificar programas educativos ${ }^{17}$.

A associação entre fatores de desenvolvimento na saúde de crianças requer o investimento na alimentação e na estimulação, como forma de proteger, prevenir e controlar defasagens no desenvolvimento infantil. O objetivo é avaliar o desenvolvimento cognitivo e motor, autocuidados, linguagem e socialização de crianças pré-escolares e sua relação com o estado nutricional.

\section{MÉTODO}

\section{Local e participantes}

O local de estudo concentrou-se na periferia da cidade de Diamantina, localizada no Alto Vale do Jequitinhonha, região que se caracteriza por ser uma das mais pobres do Estado de Minas Gerais e pelas condições insatisfatórias de vida de parcela significativa da população ${ }^{18,19}$.

Os participantes foram crianças matriculadas na instituição Casa da Criança de Diamantina, que compreende uma creche (zero a quatro anos) e uma pré-escola (cinco a seis anos), localizada em um bairro onde predominam famílias de baixa renda. Aproximadamente 100 crianças frequentavam a instituição e recebiam pelo menos três refeições no período em que estavam no local. Para amostragem ${ }^{20}$, foram sorteadas as crianças entre aquelas que frequentavam a creche e que não apresentavam problemas de saúde. Optou-se pelo sorteio de oito crianças para cada uma das seguintes faixas etárias: de 0-1 ano, 1-2 anos, 2-3 anos, 3-4 anos, 4-5 anos e 5-6 anos, totalizando 48 sujeitos.

\section{O Inventário Portage}

É um instrumento avaliativo que também permite a proposição de atividades pertinentes à estimulação e à formulação de programas infantis pré-escolares. É importante destacar 
que o guia Portage é utilizado em estudos sobre desenvolvimento ${ }^{21}$ e é aplicado em programas de acompanhamento de desenvolvimento infantil em diferentes continentes, com diversas populações.

\section{DELINEAMENTO E PROCEDIMENTOS}

As avaliações baseadas no Inventário Portage Operacionalizado englobaram o desenvolvimento cognitivo e motor, autocuidados, linguagem e socialização das crianças e as condições socioeconômicas das famílias. As medidas antropométricas das crianças foram registradas para avaliação do estado nutricional. Um questionário socioeconômico foi respondido pelos pais ou responsáveis. As avaliações foram realizadas em uma sala e as observações em outras dependências da própria instituição.

O material utilizado para as avaliações foi composto por diferentes objetos, brinquedos, jogos, que eram indicados no Inventário Portage para cada uma das faixas etárias e para cada área de desenvolvimento. De uma forma geral, o acervo conteve brinquedos que faziam parte do cotidiano das crianças, como bolas, quebra-cabeças, papel/tesoura e utensílios domésticos de brinquedo ou reais adquiridos no comércio local da própria cidade.

Semanas antes do início das avaliações, a equipe passou alguns dias entre as crianças, para que elas se habituassem e para evitar reatividade por parte dos sujeitos. A equipe de avaliadores foi constituída por dois observadores e um mediador em cada sessão de aproximadamente 30 minutos, na qual o mediador jamais insistia no prosseguimento das atividades, se a criança apresentasse sinais de cansaço.

O critério de desempenho comum para as áreas cognitiva, motora, autocuidados, linguagem e socialização envolveu a ocorrência de no mínimo três respostas corretas em quatro tentativas. Além disso, para que uma resposta fosse considerada correta, a criança deveria iniciá-la em 30 segundos (a não ser que fosse especificado de modo diferente) após o início da tentativa, caracterizada por uma instrução, modelo e/ou apresentação de um determinado objeto, conforme o caso.

Em alguns casos, à medida que se constatava que o desempenho da criança era insuficiente, ou seja, quando a criança não respondia ao estímulo dado, conforme instrução contida no Inventário Portage Operacionalizado, havia retrocesso na faixa etária.

Para tanto, foi utilizado o seguinte critério: apresentação da tarefa três vezes, se a criança obtivesse pelo menos um acerto, passava-se para a próxima tarefa. Caso a criança não conseguisse nenhum acerto mediante as três chances dadas, havia interrupção do processo e passava-se para a faixa etária anterior. Se a criança não acertasse cinco itens, retrocedia-se na faixa etária. Só foram consideradas como atividades realizadas aquelas cujas observações foram tidas como positivas para ambos observadores.

Para a avaliação das áreas de socialização e autocuidados, optou-se por entrevistar os educadores e os pais nos casos em que os educadores não conheciam a situação da criança a respeito de um determinado item. Isso se deu porque alguns itens que continham essas escalas são algumas vezes mais difíceis de observação direta e somente podiam ser bem conhecidos pelos pais e educadores.

Para a avaliação socioeconômica foi utilizado o questionário respondido pelos pais/responsável, que forneceu informações relevantes sobre as famílias das crianças, tornando possível a análise socioeconômica. Foi utilizado como instrumento de medição do nível socioeconômico um questionário para identificação de grupos vulneráveis à desnutrição infantil, especialmente elaborado para populações carentes ${ }^{22} \mathrm{e}$ adaptado do modelo original para ser utilizado com a população brasileira ${ }^{23}$.

Foi utilizado o aplicativo de informática Excel ${ }^{\circledR}$ (Microsoft Office ${ }^{\circledR}$ ) para tabulação e confecção de gráficos e o aplicativo estatís- 
tico SPSS ${ }^{\circledR}$ para Windows ${ }^{\circledR}$. A análise de dados foi de correlação (áreas de desenvolvimento e estado nutricional).

Atendendo as diretrizes éticas internacionais para pesquisas biomédicas envolvendo seres humanos, foi obtido o termo de consentimento livre e esclarecido dos pais ou representantes legais após encontro em que foram informados sobre os objetivos e procedimentos da pesquisa*. A direção da Casa da Criança foi esclarecida sobre a pesquisa e forneceu sua anuência formal para que o estudo fosse realizado na instituição.

\section{RESULTADOS}

Na faixa etária de zero a um ano de idade, verificou-se correlação significante $(\mathrm{p}<0,05)$ entre o desenvolvimento cognitivo e o motor, assim como entre a cognição e o estado nutricional. Observou-se que o déficit nutricional influiu negativamente tanto no desenvolvimento motor quanto nas habilidades cognitivas nessa faixa etária.

Na faixa etária de um a dois anos de idade, houve correlação significante $(\mathrm{p}<0,05)$ entre a linguagem e o estado nutricional, assim como foi possível verificar que peso e altura apresentaram correlação significativa entre si. Observou-se que as crianças na faixa etária de um a dois anos obtiveram resultados satisfatórios (número de acertos acima de $60 \%$ ) em todas as habilidades testadas (Tabela 1).

$\mathrm{Na}$ faixa etária de dois a três anos de idade as crianças obtiveram resultados satisfatórios em todas as habilidades avaliadas, porém vale ressaltar que na área cognitiva,

Tabela 1: Desempenho nas áreas de desenvolvimento (cognição, motricidade, autocuidados, linguagem e socialização) em porcentagem para cada faixa etária das crianças. Observação: a avaliação cognitiva das crianças do grupo com idade entre 2-3 anos foi efetuada na faixa etária de 1-2 anos

\begin{tabular}{lccccc}
\hline Crianças & Cognição(\%) & Motor(\%) & Autocuidados(\%) & Linguagem(\%) & Socialização(\%) \\
\hline 0-1 ano & $53,57^{*}$ & 58,89 & 92,30 & 38,00 & 70,71 \\
1-2 anos & 70,00 & 83,82 & 75,00 & $61,24^{*}$ & 72,50 \\
2-3 anos & 96,25 & 77,20 & 70,90 & 70,63 & 75,00 \\
3-4 anos & 63,59 & 90,83 & 77,14 & 65,48 & 53,57 \\
4-5 anos & 73,86 & 92,19 & 70,11 & 85,83 & 75,00 \\
5-6 anos & 32,95 & 84,26 & $58,89^{*}$ & 78,57 & 78,79 \\
\hline
\end{tabular}

* Correlação estatisticamente significante $(\mathrm{p}<0,05)$. Diamantina, 2005.

conforme recomendado no Inventário Portage, foi necessário retroceder à faixa etária anterior. Tal rebaixamento de faixa etária indicou que as crianças avaliadas se encontravam em acentuada defasagem nessa habilidade.

Por sua vez, na faixa etária de três a quatro anos de idade ocorreu menor percentual de comportamento na área de socializa- ção $(53,57 \%)$. Na faixa etária de quatro a cinco anos observou-se também resultado acima de $60 \%$ de acertos em todas as áreas avaliadas.

$\mathrm{Na}$ faixa etária de cinco a seis anos de idade houve correlação significativa $(\mathrm{p}<0,05)$ entre autocuidados e o estado nutricional (indicador peso/altura). Observou-se nessa faixa

* O estudo foi aprovado pelo Comitê de Ética em Pesquisa da Universidade Federal dos Vales do Jequitinhonha e Mucuri, registro no. 051/2006. 
defasagem $(<60 \%)$ na área cognitiva $(32,95 \%)$ e de autocuidados $(58,89 \%)$.

Para a faixa etária de zero a um ano de idade foi possível observar que o Inventário Portage não permite avaliação uniforme para todas as crianças compreendidas nessa fase, considerando-se que nessa faixa os comportamentos seriam mais bem compreendidos se as crianças fossem agrupadas em faixas por meses de idade. Percebeu-se que o desenvolvimento infantil está estritamente ligado ao desenvolvimento físico, o qual apresenta intensa complexidade entre zero e um ano de idade.

Verificou-se que o desenvolvimento cognitivo esteve comprometido na faixa etária de zero a um ano de idade, enquanto houve associação entre a motricidade e o estado nu- tricional nas faixas de dois a três anos (com relação ao peso) e de três a quatro anos (com relação à altura). A linguagem, por sua vez, apresentou-se deficitária na faixa de um a dois anos (com relação ao estado nutricional).

Os resultados da análise estatística para algumas áreas são apresentados na Tabela 2, em que os coeficientes negativos indicam variáveis inversamente proporcionais, enquanto os coeficientes positivos indicam variáveis diretamente relacionadas.

A análise de correlação parcial demonstrou que ocorreu correlação estatisticamente significante entre os pares de variáveis: cognição $x$ linguagem, socialização $x$ autocuidado e desenvolvimento motor $\mathrm{x}$ estado nutricional (peso/altura e peso/idade).

Tabela 2: Correlação entre o desenvolvimento cognitivo, motor e autocuidados. Os dados representam o coeficiente de correlação com o p valor entre parênteses.

\begin{tabular}{lcccc}
\hline & WHP (P/A) & WAP $(\mathrm{A} / \mathrm{I})$ & Linguagem & Socialização \\
\hline Cognição & $-0,863(0,688)$ & $0,0045(0,983)$ & $0,5196(0,009)^{*}$ & $-0,0499(0,835)$ \\
Motor & $-0,4284(0,037)^{*}$ & $-0,4408(0,031)^{*}$ & $-0,1495(0,486)$ & $-0,0875(0,684)$ \\
Autocuidado & $-0,1372(0,523)$ & $-0,1354(0,528)$ & $0,0405(0,851)$ & $0,4331(0,035)$ \\
\hline
\end{tabular}

* Significância estatística $(\mathrm{p}<0,05)$. Diamantina, 2005.

\section{DISCUSSÃO}

A adaptação do Inventário Portage no presente estudo possibilitou a observação das associações entre as áreas do desenvolvimento e o estado nutricional infantil de crianças brasileiras. Embora o Inventário tenha sido elaborado originalmente nos Estados Unidos, foi possível sua aplicação na realidade brasileira. Estudo comparativo ${ }^{24}$ entre crianças brasileiras e norte-americanas utilizou a nomeação de figuras, familiaridade com o conceito representado e a complexidade visual. Os autores verificaram que o conjunto de figuras utilizado se mostrou adequado para uso em diferentes culturas, embora tenham recomendado evitar o uso de algumas figuras que pu- dessem produzir inconsistência de nomeação. Assim sendo, a adaptação procedida no presente estudo buscou evitar tais tipos de inconsistências nominais.

Sobre a mediação no estudo, vale ressaltar que o desempenho das crianças foi aquele observado espontaneamente diante de uma pessoa familiar, e o avaliador pôde ir obtendo medidas de linha-de-base do próprio desempenho do mediador. Nesse sentido, coube ao observador orientar o mediador em como apresentar as condições à criança, além de observar e registrar o comportamento apresentado. Esse procedimento permitiu que o Inventário Portage fosse utilizado com facilidade frente à realidade local e, além disso, a adequação dos brinquedos utilizados foi de grande relevância 
para que fossem semelhantes àqueles disponíveis no ambiente das crianças.

Um estudo que utilizou o Inventário Portage foi realizado satisfatoriamente na Espanha $^{17}$ e consistiu-se na avaliação do desenvolvimento psicológico de crianças de um a cinco anos de idade. Enquanto que naquele estudo considerou-se a relação do abandono físico no desenvolvimento infantil, neste levouse em conta a relação com o estado nutricional de crianças de famílias de baixa renda. A condição de abandono físico esteve associada ao atraso no desenvolvimento infantil, sendo que sugeriram futura comparação com os estilos de interação entre crianças e seus pais/cuidadores.

No presente trabalho, verificou-se que o desenvolvimento cognitivo, a linguagem e os autocuidados apresentaram relação com o estado nutricional das crianças, estando, por sua vez, associados à condição socioeconômica das famílias. Porém, em algumas faixas etárias, foi possível perceber que também os estilos de interação entre as crianças e suas famílias precisam ser mais bem investigados.

$\mathrm{O}$ desenvolvimento da linguagem, por sua vez, apresenta estreita ligação com a exploração do ambiente e com a interação social. Conforme demonstrado em um estudo recen$t^{25}$ com a interação educadora/bebê, existe um desafio para os educadores em relação ao cuidado simultâneo de várias crianças em diferentes faixas etárias. A responsividade interpessoal das crianças foi investigada, enquanto processo de comunicação entre cuidador e criança no ambiente de uma creche, e os resultados mostraram que a qualidade da interação social e, consequentemente, o desenvolvimento da linguagem parecem depender da quantidade de crianças por cuidador/educador.

Considerando a grande variação na apresentação de habilidades pelas crianças na faixa etária de zero a um ano de idade (cognição, motricidade, autocuidado, linguagem e socialização), optou-se por agir com cautela na avaliação de tal faixa etária na análise final. Suge- re-se que em outro estudo possa ocorrer a investigação detalhada, de forma que as crianças venham a ser agrupadas em períodos mais curtos nessa faixa etária, como de três em três meses, o que permitiria avaliação das referidas áreas de desenvolvimento. Futuramente, a utilização de testes para crianças nessa faixa deve então levar em conta intervalos mais curtos medidos em meses ao invés de anos.

Quanto ao desenvolvimento motor, é interessante observar que ele melhora a partir do momento em que as crianças passam a frequentar uma creche, entretanto alguns autores colocam em questão se isso ocorre devido exclusivamente à família, à creche ou a ambos ${ }^{26}$. Creche de boa qualidade pode ser uma excelente oportunidade para as crianças estarem em um local seguro, onde possam brincar, além de se alimentarem satisfatoriamente e terem companhia de outras da mesma idade.

Sobre a interação ambiental, é fundamental a identificação dos fatores de risco que parecem estar associados à qualidade do ambiente e a algumas características das crianças, contribuindo como um aporte ao estudo do desenvolvimento infantil no que diz respeito a grupos vulneráveis, priorizando as ações protetoras de saúde ${ }^{27}$. Os aspectos preventivos devem ser investigados, incluindo programas de promoção de saúde e qualidade de vida das pessoas, em especial da população infantil. Com a identificação de variáveis associadas ao desenvolvimento infantil, é possível programar ações orientadas para as principais necessidades das crianças.

A importância da estimulação para o desenvolvimento cognitivo infantil, da melhoria das condições materiais e da dinâmica familiar, parte do efeito da estimulação sobre a cognição, que é mediada pela condição materna de trabalho e de seu nível de escolarida$\mathrm{de}^{28}$. Embora não tenha sido o objeto direto deste estudo, verificou-se que a formação dos cuidadores é limitada para a promoção adequada e estimulação das crianças 
frequentadoras da creche. Além disso, o número insuficiente de profissionais de educação na instituição, devido à sua restrição orçamentária, faz com que haja número excessivo de crianças por cuidador, o que prejudica a interação entre eles.

Assim, com relação à população alvo, é urgente a priorização de programas que protejam a saúde, com atenção especial às famílias e às creches para adoção de modos de vida saudáveis, além da identificação de situações de risco. Consideramos que a qualidade do ambiente e da relação cuidadorcriança é fundamental para o pleno desenvolvimento infantil, e devem ser estimuladas e promovidas ações educativas e de formação voltadas aos educadores e familiares das crianças. Dessa forma, recomendase também a inclusão de práticas de estimulação cotidiana e a promoção de hábitos saudáveis nas atividades desenvolvidas nas creches, com participação de educadores e familiares.

O estudo permitiu investigar associações significativas entre algumas áreas de desenvolvimento e o estado nutricional, como o observado na faixa etária de um a dois anos (linguagem e estado nutricional) e de cinco a seis anos (autocuidados e estado nutricional, e linguagem e altura).

\section{REFERÊNCIAS}

1. Valente FLS. Do combate à fome à segurança alimentar e nutricional: o direito à alimentação adequada. Rev Nutr. 1997;10(1):20-36.

2. Wasantwisut E. Nutrition and development: other micronutrients' effect on growth and cognition. Southeast Asian J Trop Med Public Health. 1997;28(2):78-82.
A avaliação das áreas do desenvolvimento infantil por faixa etária possibilitou revelar que o desenvolvimento cognitivo foi comprometido na faixa etária de zero a um ano de idade, enquanto houve associação entre a motricidade e o estado nutricional nas faixas de dois a três anos (com relação ao peso) e de três a quatro anos (com relação à altura). A linguagem, por sua vez, apresentou-se deficitária na faixa de um a dois anos (com relação ao estado nutricional).

As implicações do Inventário Portage para o tratamento e a intervenção são importantes para a manutenção das interações adequadas entre as crianças e pais/cuidadores. As ações devem ser dirigidas precisamente para o enriquecimento de tais interações mediante planos formativos, que possam incrementar o repertório dos educadores e das famílias das crianças que frequentam creches.

\section{AGRADECIMENTOS}

À direção da Casa da Criança de Diamantina $(\mathrm{MG})$, às mediadoras Aline $\mathrm{F}$. Miranda e Marciane Almeida e aos colaboradores Josefina Murta, M.A.B., Rafael C. Teixeira e Valéria M. M. Franco. Especial agradecimento a Juliana V. M. Mambrini pelo apoio estatístico.

3. Black RE. Informe de la conferencia: El cinc para la salud infantil. Organización Pan-Americana de Salud - NutriInfo 4; 1998. p. 1-6.

4. Martins IS, Alvarenga AT, Siqueira AAF, Szarfarc SC, Lima FD. As determinações biológicas e sociais da doença: um estudo da anemia ferropriva. Rev Saude Publica. 1987;21(2):73-89. 
5. Ruz M, Cavan KR, Bettger WJ, Thompson L, Berry M, Gibson RS. Development of a dietary model for the study of mild zinc deficiency in humans and evaluation of some biochemical and functional indices of zinc status. Am $J$ Clin Nutr. 1991;53:1295-303.

6. Gibson RS. (1989) A growth-limiting, mild zinc-deficiency syndrome in some Southern Ontario boys with low height percentiles. Am J Clin Nutr. 1989;49:1266-73.

7. Assis AMO, Prado MS, Freitas MCS, Cruz MM. Deficiência de vitamina A e desnutrição energético protéica em crianças de localidades do semi-árido baiano. Rev Nutr. 1997;10(1):70-8.

8. Organização Pan-Americana de Saúde. Bases para el desarrollo del documento de posición y para el plan de acción regional para la obesidad en la pobreza. NutriInfo 1; 1995.

9. Reed BA, Habicht JP, Niameogo C. The effects of maternal education on child nutritional status depend on socioenvironmental conditions. Int J Epidemiol. 1996;25(3):585-92.

10. Brown JL, Pollitt E. Malnutrition, poverty and intellectual development. Sci Am. February, 1996. p. 26-31.

11. Pollitt E. Timing and vulnerability in research on malnutrition and cognition. Nutr Rev. 1996;54(2):S49-5.

12. UNICEF. Fundo das Nações Unidas para a Infância. Situação mundial da infância. 1998. p. 132.

13. Baddeley A, Meedks Gardner J, Grantham-McGregor SM. Cross-cultural cognition: developing tests for developing countries. Appl Cogn Psychol. 1995;9(S):173-95.

14. Grantham-McGregor SM, Walker SP, Chang SM, Powell CA. (1997) Effects of early childhood supplementation with and without stimulation on later development in stunted Jamaican children. Am J Nutr. 66(2): 247-253.
15. Meeks Gardner JM, Grantham-McGregor SM, Chang SM, Himes JH, Powell CA. Activity and behavioral development in stunted and nonstunted children and response to nutritional supplementation. Child Development. 1995;66:1785-97.

16. Bluma S, Shearer M, Frohman A, Hilliard J. Guia Portage de educacion pré-escolar: manual de entrenamiento. Portage, Wisconsin: Cooperative Educational Service Agency. 1978.

17. Osuna MJP, Cabrera JH, Morales MCM. Estudio de las consecuencias del abandono físico en el desarrollo psicológico de niños de edad preescolar en España. Chile Abuse Neglect. 2000;24(7):911-24.

18. Fundação João Pinheiro. Atenção Básica à Saúde em Minas Gerais. Belo Horizonte: Centro de Estudos Econômicos e Sociais; 2003. p. 151.

19. Souza JVA. Luzes e sombras sobre a história e a cultura do Vale do Jequitinhonha. Trabalho, cultura e sociedade no norte/ nordeste de Minas. Montes Claros: Best Comunicação e Marketing; 1997. p. 144.

20. Lwanga SK, Lemenshow S. Sample size determination in health studies: a practical manual. World Health Organization WHO, Geneva, 1991. p. 82.

21. Williams LCA, Aiello ALR. O Inventário Portage Operacionalizado: intervenção com famílias. São Paulo: Editora Memnon; 2001. p. 299.

22. Issler RMS, Giugliani ERJ. Identificação de grupos mais vulneráveis à desnutrição infantil pela medição do nível de pobreza. J Pediatr. 1997;73(2):101-5.

23. Alvarez ML, Wurgaft F, Salazr ME. Mediciones del nivel socioeconómico bajo urbano en familias con lactante desnutrido. Arch Latinoam Nutr. 1989;32(4):650-62.

24. Miranda MC, Pompéia S, Bueno OFA. Um estudo comparativo das normas de um conjunto de 400 figuras entre crianças brasileiras e americanas. Rev Bras Psiquiatr. 2004;26(4):226-33. 
25. Bressani, MCL, Bosa CA, Lopes RS. A responsividade educadora-bebê em um berçário: um estudo exploratório. Rev Bras Crescimento Desenvolv Hum. 2007;17(3):21-36.

26. Rezende MA, Beteli VC, Santos JLF. Follow-up of the child's motor abilities in day-care centers and pre-schools. Rev Lat Am Enfermagem. 2005;13(5):619-25.

27. Martins MFD, Costa JSD, Saforcada ET, Cunha MDC. Qualidade do ambiente e fatores associados: um estudo em crian- ças de Pelotas, Rio Grande do Sul, Brasil. Cad Saude Publica. 2004;20(3):710-8.

28. Andrade SA, Santos DN, Bastos AC, Pedromônico MRM, Almeida-Filho N, Barreto ML. Ambiente familiar e desenvolvimento cognitivo infantil: uma abordagem epidemiológica. Rev Saude Publica. 2005;39(4):606-11.

Recebido em: 10/jul./2010

Modificado em 21/nov./2010 Aceito em 16/jan./2011 